\section{Gender reassignment today}

SIR,-Mr Grant Williams's letter (12 September, p 671) protesting about gender reassignment surgery requires a reply. He is unaware of studies done in our department, the results of which were presented at a conference in December 1986 at this hospital. We presented the results of a retrospective study of 150 patients and a second, randomised controlled study of 40 patients. ${ }^{12}$ We studied 150 male transsexuals at different stages of treatment: assessment stage $(n=50)$; waiting list stage $(n=50)$; and postoperative stage $(n=50)$. The results indicated a significant reduction in neurotic symptoms and improved social state in transsexuals postoperatively compared with patients at the waiting list stage, who fulfilled the criteria for surgery but were awaiting operation, and assignment to an assessment group.

The second study compared two groups of male transsexuals who had been assessed and carefully selected for surgery. They were randomly assigned to one of two groups; the experimental group had their waiting time for surgery brought forward so that they were operated on within three months of fulfilling the criteria, and the control group had to wait for the routine two years before undergoing surgery. All the patients were equally matched for age, social class, number of years of clinic attendance, and several other variables that might affect outcome. The results suggested that after two years of follow up there were significant differences between the two groups on a number of psychiatric and social variables, showing a significant advantage for the experimental group over the controls.

Of the many male transsexuals operated on since 1984 , not a single patient has requested shortening of the distal limbs. The most common surgical request, other than gender reassignment, is for thyroid cartilage reduction, and a minority request breast augmentation, a procedure which we discourage patients from pursuing.

Three major independent reviews have suggested that treatment produces unsatisfactory results or failure in only $10-15 \%$ of cases and that most patients who have gender reassignment surgery have a satisfactory outcome. ${ }^{3-5}$ A study by Robin $e t$ al suggested that $85 \%$ of male transsexuals who had undergone operation reported improvement in their condition, and none of the patients regretted the operation, including the $15 \%$ of patients who had surgical complications that were later corrected. ${ }^{6}$

In the early stages of assessment it is not uncommon to be presented with various patients with gender identity problems, including transvestites and effeminate homosexuals. The assessment of new patients includes screening for hormone concentrations and tests for evidence of infection with hepatitis B and the human immunodeficiency virus. Not surprisingly, a very small percentage of such patients show evidence of past infection with hepatitis B. Since 1984 no carriers of hepatitis B have received gender reassignment surgery.

Many of the patients who are referred are not classified as transsexuals. Those that are diagnosed as transsexuals have to fulfil strict criteria before being referred for gender reassignment surgery. We see 175-200 new patients a year and refer 30-40 a year for surgery, some years much fewer.

Transsexualism and severe gender dysphoria have all the characteristics of severe psychological disturbance associated with a great deal of suffering, which we have found to be much relieved by gender reassignment surgery in carefully selected patients. The opportunity to study and treat severe gender dysphoria and severe gender identity disorders is a recent development. Such studies may lead to a better understanding of other disorders of gender identity and other types of pathological sexual behaviour, many of which cause great concern and distress in society today.

Those who fail adequately to integrate the contra-gender aspect of themselves into their total personality and suffer from severe gender dysphoria or gender identity strain deserve our concern, help, and scientific interest.

Charles Mate-Kole DON MONTGOMERY JAMES DALRYMPLE STEVEN HIRSCH

\section{Gender Identity Clinic}

and Academic Department of Psychiatry,

Charing Cross and Westminster Medical School,

London W6 8RP

1 Mate-Kole C, Freschi M, Robin A. Aspects of psychiatric symptomatology at different stages in the treatment of transsexualism. Br f Pychiatry (in press)

2 Mate-Kole C, Freschi M. Psychiatric aspects of sex reassignmen surgery. Brf Hosp Med (in press).

Lundstrom B, Pauly I, Walinder J. Outcome of sex reassignmen surgery. Acta Psychiatr Scand 1984;70:289-94.

4 Pauly. I. Outcome of sex reassignment surgery for transsexuals. Aust NZ Y Psychiatry 1981;15:45-51

5 Lothstein L. Sex reassignment surgery: historical, bioethical and theoretical issues. Am $\mathcal{Y}$ Psychiatry 1982;139:417-26.

6 Robin A, Mate-Kole C, Freschi M. The benefits of surgical reassignment as perceived by operated male transsexuals. In Mate-Kole C, ed. The state of transsexualism - proceedings of th international conference on gender identity. London: Schering Health Care, 1987.

\section{District cancer physicians: integration or fragmentation?}

SIR,-Dr L J Donaldson's leading article (19 September, $p$ 682) criticises the report of the working party of the Association of Cancer Physicians on the subject of district cancer physicians on the grounds that its proposals do not imply an integration of services. ${ }^{1}$ The type of integration that he seems to prefer is that where cancer services are centred in the subregional centres to the exclusion of any activity in district general hospitals.

The fallacy of this argument is shown by the existing pattern of cancer treatment based on radiotherapy centres, which are of necessity organised so that they are centrally placed to achieve the maximum efficiency of the capital investment in equipment. The problems that this causes for the patient with cancer are clear from the report; patients in many health districts receive their treatment at some distance from their home with financial consequences to themselves and to the health authority and potential medical con sequences when complications of the disease and its treatment arise. The alternative to centralised treatment is treatment by non-experts in the district general hospital. That this is something that happens even in the health region over whose clinical policy Dr Donaldson presides has been shown by Woll. ${ }^{2}$ The current medical management of patients with cancer is therefore either conducted at a great distance or fragmented.

The appointment of district cancer physicians would facilitate the integration Dr Donaldson seeks by ensuring that those patients who are treated with chemotherapy in their local hospital are managed by someone with the necessary skill. The need for this is illustrated by the work of gynaecologists; although they have considerable training in the management of diabetes or heart disease in pregnancy, they have no hesitation in referring patients to the appropriate physician, whereas when a patient requires chemotherapy for carcinoma of the ovary with drugs with a very low therapeutic index they have to take this on them- selves. The second integrative function is in the liaison with the radiotherapy unit. The current pattern of weekly visits by the radiotherapist does not provide the best service for managing a patient with cancer. A patient may have a small cell carcinoma of the bronchus diagnosed one week and then wait seven days for the radiotherapist to see him and a further week for admission to the radiotherapy centre, during which time he may deteriorate from an eminently treatable state to being moribund. The appointment of a district cancer physician would ensure that the radiotherapist's time would be spent in dealing with genuine radiotherapeutic problems.

In his penultimate paragraph Dr Donaldson argues that the role of the district cancer physician in integrating the acute cancer treatment services with the supportive care role would introduce too great a rigidity. This seems to be a complete negation of his basic premise.

S M CRAWFORD

Clinical Oncology Unit,

University of Bradford,

West Yorkshire BD7 IDP

1 MacIllmurray MB. District cancer physicians. Report of the working group of the Association of Cancer Physicians. $\mathcal{F} R$ Coll Physicians Lond 1987;21:117-21.

2 Woll PJ. Who treats cancer? $f(R$ Coll Physicians Lond 1987;21:61-6.

Is schizophrenia a neurodevelopmental disorder?

SIR,-Drs Robin M Murray and Shôn W Lewis (19 September, p 681) address the issue of obstetric complications and potential neurodevelopmental deviance as a risk factor for schizophrenia, particularly in patients characterised by enlargement of the cerebral ventricles and cortical sulci. We wonder if their arguments might be extended not only to vulnerability to the disorder itself but also to the different patterns of response to its treatment with neuroleptic agents.

The major clinical, ethical, and potentially legal issue in the long term treatment of schizophrenia with such drugs remains the emergence of involuntary movements (tardive dyskinesia) in a variable proportion of patients. ${ }^{1}$ We have recently found that schizophrenic patients with tardive dyskinesia are characterised by an excess release of developmental (primitive/neonatal) reflexes. ${ }^{2}$ These signs, typified by the grasp, snout, corneomandibular, and glabellar reflexes, are readily released in the fetus and neonate but lost in the process of unhindered neuronal maturation and myelination that leads to normal adulthood. ${ }^{3}$ As their subsequent reappearance is usually associated with cortical or diffuse cerebral dysfunction ${ }^{2}$ such data add weight to the increasing body of evidence that signs of "organicity" are particularly associated with, and may predispose to, tardive dyskinesia. The excess release of developmental reflexes in schizophrenic patients with this movement disorder is perhaps suggestive of anomalies in brain development rather than cerebral deterioration from a once normal level of functioning.

Additionally, Drs Murray and Lewis note the greater vulnerability of the male brain to early damage. We have also found a greater severity of tardive dyskinesia among male schizophrenic patients affected by this movement disorder. ${ }^{4} \mathrm{~A}$ recent study has confirmed that neonatal periventricular haemorrhage, considered by Drs Murray and Lewis to be a potential cause of neurodevelopmental deviance, is more common in boys when pregnancy is complicated by factors such as prematurity and low birth weight. ${ }^{5}$

Perhaps anomalies in brain development may predispose not only to schizophrenia but also to 